\title{
НЕСМЕЩЕННАЯ ОЦЕНКА ПАРАМЕТРА ПРИ ЦЕНЗУРИРОВАННЫХ НАБЛЮДЕНИЯХ
}

V. OLMAN. PARAMEETRI NIHUTAMATA HINNANG TSENSEERITUD VAATLUSTE KORRAL V. OLMAN. UNBASED PARAMETER ESTIMATION UNDER CENSORED OBSERVATIONS

(Представил Н. Алумяэ)

Рассмотрим следующую статистическую модель. Над случайной величиной $X$ проводится $n>1$ независимых экспериментов. Известна функция распределения $F(x, \lambda)$ величины $X$ с точностью до параметра $\lambda \Subset \Lambda$, где $\Lambda$ - множество возможных значений параметра $\lambda$. При цензурировании наблюдений указывается лишь, с какой стороны от заданной точќи $a$ лежит реализация случайной величины. Задача заключается в несмещенном оценивании неизвестного параметра $\lambda$ по peзультатам экспериментов. В качестве такой оценки рассмотрим $\hat{\lambda}$, являющуюся решением уравнения

$$
k / n=F(a, \hat{\lambda})
$$

где $k$ - число реализащий, лежащих слева от $a$. Очевидны следующие требования к числу $a$ :

1) $a \in \bar{S}_{\lambda}$, где $S_{\lambda}=\left\{a \in R_{1}: 0<F(a, \lambda)<1\right\}, \lambda-$ истинное значение оцениваемого параметра, $\bar{S}_{\lambda}$ - замыкание множества $S_{\lambda}$;

$2)$ из равенства $F\left(a, \lambda_{1}\right)=F\left(a, \lambda_{2}\right), \quad a \in \bar{S}_{\lambda_{1}} \cap \bar{S}_{\lambda_{2}}$ должно следовать $\lambda_{1}=\lambda_{2}$, иначе эти два распределения будут неразличимы с точки зрения имеющейся информации;

3) функция $F(a, \lambda)$ непрерывна по $\lambda$, и множество значений $F(a, \lambda)$ при $\lambda \in \Lambda$ совпадает со множеством $[0,1]$.

Перечисленные условия являются достаточными для существования и единственности решения уравнения (1) относительно $\hat{\lambda}$.

Т ео ре м а. Если выполнены условия 1)-3), то решение уравнения (1) является несмещенной оценкой параметра $\lambda$ при фиксированном $a \in S_{\lambda}$ тогда и только тогда, когда

$$
F(a, \lambda)=\lambda c+l,
$$

где $l$ и с - произвольные веществвнные числа такие, что $0 \leqslant \lambda c+l \leqslant 1$ для всех $\lambda$ таких, ито $S_{\lambda} \ni a$.

Доказательство. Пусть $\hat{\lambda}=G(k / n, a)$ - решение уравнения (1), т. е. $F(a, G(k / n, a))=k / n$. Тогда в силу несмещенности оценки $\hat{\lambda}$ и того, что случайная величина $k$ распределена по закону Бернулли, для $a \in S_{\lambda}$ получим

$$
\sum_{k=0}^{n} G(k / n, a) C_{n}^{k} F^{k}(a, \lambda)(1-F(a, \lambda))^{n-k_{i}}=\lambda .
$$


Выберем $\lambda_{r}=G(r / n, a), r=0,1,2, \ldots, n, a \in \bar{S}_{\lambda_{r}}$ и перепишем уравнение (2) в виде

$$
\begin{aligned}
\sum_{k=0}^{n} G(k / n, a) C_{n}^{k}(r / n)^{k}(1-r / n)^{n-k} & =G(r / n, a), \\
r & =0,1, \ldots, n .
\end{aligned}
$$

Равенства (3) представляют собой линейную систему относительно $\{G(k / n, a)\}^{n}{ }_{k=0}$. Решение системы (3) эквивалентно определению всех собственных векторов матрицы $A=\left\{C^{k}{ }_{n}(r / n)^{k}(1-r / n)^{n-k}\right\}, \quad k, r=$ $=0,1,2, \ldots, n$, соответствующих собственному числу, равному 1 . Заметим, что первой строкой матрицы $A$ является $(1,0 \ldots, 0)$, а последней - $(0,0, \ldots, 0,1)$. Следовательно, 1 является характеристическим числом кратности по крайней мере 2. Остальные собственные числа матрицы $A$ совпадают с собственными числами матрицы

$$
A^{*}=\left\{C_{n}^{k}(r / n)^{k}(1-r / n)^{n-k}\right\}, \quad k, n=1,2, \ldots, n-1 .
$$

Но так как

$$
\sum_{k=0}^{n} C_{n}^{k}(r / n)^{k}(1-r / n)^{n-k_{1}}=1, \quad r=0,1,2, \ldots, n
$$

то $\max _{1 \leqslant r \leqslant n-1} \sum_{k=1}^{n-1} C_{n}^{k}(r / n)^{k}(1-r / n)^{n-k}<1$, откуда по теореме Фробениуса

[1] следует, что максимальное собственное число матрицы $A^{*}$ меньше 1. Таким образом, кратность собственного числа 1 у матрицы $A$ равна 2. Найдем соответствующие ему собственные векторы р и q. Очевидно, вектор $\mathrm{p}=(1,1, \ldots, 1)$ собственный в силу (4), а вектор $q=(0,1 / n, 2 / n, \ldots, n / n)$ собственный в силу равенства

$$
\sum_{k=0}^{n} C_{n}^{k} t^{k}(1-t)^{n-k} k=n t, \quad 1 \geqslant t \geqslant 0 .
$$

Так как р и q линейно независимы, то все решения системы (3) описываются линейными комбинациями векторов р и q, т. е.

$$
G(r / n, a)=c^{-1} r / n-l / c, \quad r=0,1,2, \ldots, n,
$$

где $c$ и $l$ - произвольные постоянные. Подставляя (6) в (2), получаем

$$
\sum_{k=0}^{n}\left(c^{-1} k / n-l / c\right) C_{n}^{k} F^{k}(a, \lambda)(1-F(a, \lambda))^{n-k}=\lambda,
$$

а используя (4) и (5), имеем

$$
F(a, \lambda) / c-l / c=\lambda,
$$

или

$$
F(a, \lambda)=c \lambda+l,
$$

что и доказывает необходимость. Достаточность легко проверить с помощью равенств (4) и (5).

Пример.

$$
F(x, \lambda)=\left\{\begin{array}{ccc}
(x-\lambda) / \sigma & \text { при } & \lambda \leqslant x \leqslant \lambda+\sigma \\
0 & \text { при } & x<\lambda \\
1 & \text { при } & x>\lambda+\sigma
\end{array}\right.
$$

т. e. $c=-1 / \sigma, l=x / \sigma$. 
В заключение вычислим дисперсию описанной несмещенной оценки. Так как

$$
D(\hat{\lambda})=\sum_{k=0}^{n} G^{2}(k / n, a) C_{n}^{k} F(a, \hat{\lambda})^{k}(1-F(a, \hat{\lambda}))^{n-k}-\lambda^{2},
$$

то, используя равенство

$$
\sum_{k=0}^{n} k^{2} C_{n}^{k} t^{k}(1-t)^{n-k}=n t[(n-1) t+1], \quad 0 \leqslant t \leqslant 1,
$$

получаем

$$
D(\hat{\lambda})=n^{-1}(1-c \lambda-l)(c \lambda+l) / c^{2},
$$

т. е. по порядку малости дисперсии оценки $\hat{\lambda}$ совпадают с эффективными оценками.

\section{Л ИТ Е Р А Т Р А}

1 Р. а о С. Р., Линейные статистические методы и их применения, М., «Наука», 1968.

Институт кибернетики

Академии наук Эстонской ССР
Поступила в редакцию 22/II 1982

\section{УДК $535.41: 517.94$}

К решению одномерных волновых уравнений. Кард П. - Изв. АН ЭстССР, Физика * Математика, 1982, т. 31, № 3, с. 241-248 (рез. эст., англ.)

Если функция $\Theta(G)$ удовлетворяет уравнению

$$
d^{2} \Theta / d G^{2}+\left[k^{2} h^{2} N^{2}(G)+\left(4 \Phi^{2}\right)^{-1}\left(\Phi^{\prime 2}-2 \Phi \Phi^{\prime \prime} \mp g^{-2}\right)\right] \Theta=0
$$

при заданных функциях $N(G)$ и $\Phi(G)$ и постоянных $k, h, g$, то решение одномерного волнового уравнения

$$
d^{2} U / d z^{2}+k^{2} g^{2} N^{2}(G) \Phi^{2}(G)(a z / h+b)^{-2}(c z / h+d)^{-2} U=0
$$

нли

$$
d^{2} U / d z^{2}+4 k^{2} g^{2} N^{2}(G) \Phi^{2}(G)\left[\left(a^{2}+c^{2}\right) z^{2} / h^{2}+2(a b+c d) z / h+\left(b^{2}+d^{2}\right)\right]^{-2} U=0
$$

(первое в случае верхнего знака в (1), второе в случае нижнего), причем $a d-b c=1$, выражается формулой

$$
\text { или }
$$$$
U=(a z / h+b)^{1 / 2}(c z / h+d)^{1 / 2} \Phi^{-1 / 2 \Theta}
$$

$$
U=\left[\left(a^{2}+c^{2}\right) z^{2} / h^{2}+2(a b+c d) z / h+\left(b^{2}+d^{2}\right)\right]^{1 / 2} \Phi^{-1 / 2} \Theta
$$

соответственно. Параметр $G$ связан с координатой $z$ формулой

$$
\int \Phi^{-1} d G=M+g \ln \left(\frac{a z / h+b}{c z / h+d}\right)
$$

или

$$
\int \Phi^{-1} d G=M+2 g \arctan \left(\frac{a z / h+b}{c z / h+d}\right)
$$

соответственно. Приведены четыре примера одномерных волновых уравнений, решаемых в замкнутом внде этим методом. Бнбл. 2 назв. 